\title{
Low-dose computerized tomography in lung cancer screening
}

\begin{abstract}
Lung cancer screening has been a passionately debated topic since the late 1990s. Five-year survival is $53.5 \%, 26.1 \%$, and $3.9 \%$ when cancer is confined to the lung at the time of diagnosis, when there is regional nodal involvement, and when there is distant metastasis, respectively. The goal of lung cancer screening (LCS) is to shift the timing of the diagnosis to an earlier point, thus, the disease is localized to the lung, and then appropriate treatment can reduce the mortality of lung cancer. Study results from several lung cancer screening trials worldwide, including the United States, Japan, the Netherlands, Denmark, and Italy demonstrated that low-dose computerized tomography (LDCT) scanner used in LCS can increase the detection rate of lung cancer at an earlier stage. At the time of screening, the information about smoking cessation should be provided to all current smokers, while the multidisciplinary clinic affords a second opportunity to counsel patients about the benefits of quitting smoking. After two rounds of screening, there are fewer false positives as a result of comparison with the baseline screening CT that may reveal two years of pulmonary nodule stability. Decreasing the number of false -positive lung cancer screens is an area for future research. Genetic profiles and the results of the baseline screening examination can potentiate further refining the risk modeling. Risk modeling could define the frequency of follow-up in addition to who should be screened. In conclusion, LCS with LDCT has shown that there are indolent lung cancers that may not be fatal. Further studies are urgently needed if the maximization of the risk-benefit ratio in LCS has to be achieved.
\end{abstract}

Volume 6 Issue 4 - 2019

\section{Attapon Cheepsattayakorn, ${ }^{1,2}$ Ruangrong Cheepsattayakorn $^{3}$}

' $10^{\text {th }}$ Zonal Tuberculosis and Chest Disease Center, Chiang Mai, Thailand

${ }^{2}$ Faculty of Public Health, St. Theresa International College, Nakorn Nayork, Thailand

${ }^{3}$ Department of Pathology, Faculty of Medicine, Chiang Mai University, Chiang Mai,Thailand

\author{
Correspondence: Attapon Cheepsattayakorn, I $0^{\text {th }}$ Zonal \\ Tuberculosis and Chest Disease Center, 143 Sridornchai Road \\ Changklan Muang Chiang Mai 50100, Thailand Tel+ 6653 \\ |40767/66 53 276364, Fax+ 6653 |40773, 66-53-273590, \\ Email Attapon195@gmail.com
}

Received: October 19,2019 | Published: November 19, 2019

Keywords: lung cancer, screening, low-dose computerized tomography

Abbreviations: ACCP, american college of chest physicians; ASCO, american society of clinical oncology; CT, computed tomography; LDCT, low-dose computerized tomography; LCS, lung cancer screening; LLP, liverpool lung project; NCCN, national comprehensive cancer network; NLST, national lung screening trial

\section{Introduction}

In the late 1990 s, lung cancer screening has been a passionately debated topic, including the Early Lung Cancer Action Program and screening programs in Japan. ${ }^{1-4}$ Promoting cigarette smoking cessation is necessary due to a much higher risk in smokers although lung cancer does occur in non-smokers. For the decade from 2000 to 2010 , the relative risk of death from lung cancer in men and women who are current smokers, compared to men and women who are nonsmokers, were 24.97 and 25.66 , respectively. ${ }^{5}$ Five-year survival is $53.5 \%, 26.1 \%$, and $3.9 \%$ when cancer is confined to the lung at the time of diagnosis, when there is regional nodal involvement, and when there is distant metastasis, respectively. ${ }^{6}$ The goal of lung cancer screening (LCS) is to shift the timing of the diagnosis to an earlier point, thus, the disease is localized to the lung, and then appropriate treatment can reduce the mortality of lung cancer. ${ }^{6}$ Study results from several lung cancer screening trials worldwide, including the United States, Japan, the Netherlands, Denmark, and Italy demonstrated that low-dose computerized tomography (LDCT) scanner used in LCS can increase the detection rate of lung cancer at an earlier stage. ${ }^{1-4,7-9}$

\section{Lung cancer screening guidelines}

Growing list of organizations have issued guidelines for lung cancer screening with LDCT, including American Lung Association (http://www.lung.org), the National Comprehensive Cancer Network (NCCN) (http://www.nccn.org), the American Association for Thoracic Surgery, and the American Cancer Society (http://www. cancer.org), widely following the eligibility criteria and structure of the National Lung Screening Trial (NLST). ${ }^{10-12}$ The joint statement of the American College of Chest Physicians (ACCP) and the American Society of Clinical Oncology (ASCO) warned that screening should occur only in settings that can deliver the comprehensive care provided to NLST subjects. ${ }^{13}$ The American Cancer Society recommended that clinicians with access to high-volume, high-quality lung cancer screening and treatment centers should initiate a discussion about screening with apparently healthy patients aged 55 years to 74 years who have at least a 30-pack-year smoking history and who currently smoke or have quit within the past 15 years before any decision is made to initiate LCS. ${ }^{11}$

\section{Management of the patients}

The patients with positive findings, either pulmonary nodules or incidental findings is best cared for within a multidisciplinary clinic although $\mathrm{CT}$ screening for lung cancer may be effectively performed within the framework of a diagnostic radiology department. The clinic should include representatives from several medical specialties, including pulmonology, thoracic surgery, cardiology, interventional radiology, and smoking cessation that will allow suitable pulmonary nodule follow-up. Pulmonary nodule follow-up should be performed following established guidelines, such as those developed by the NCCN, for solid, semi-solid, and ground glass nodules. At the time of screening, the information about smoking cessation should be provided to all current smokers, while the multidisciplinary clinic 
affords a second opportunity to counsel patients about the benefits of quitting smoking. If a pulmonary nodule is identified to be malignant at biopsy, then further assessment by a multidisciplinary clinic can allow the patient to make an informed decision about medical care. ${ }^{14}$

\section{Obstacles to lung cancer screening with low-dose computed tomography}

The mortality benefit of LCS can only be realized if persons at risk actually participate in the LCS program. Concerns about radiation effects and discomfort of LCS process are related to reluctance to undergo screening whereas financial costs are a large obstacles to screening, particularly when downstream costs are considered. ${ }^{14}$ The primary concern with the radiation dose from computed tomography (CT) screening is the possibility of radiation-induced carcinogenesis. ${ }^{15,16}$ Subsequent follow-up examinations to work up positive findings lead to the patient's lifetime exposure although performing at low dose $\mathrm{CT}$ in the initial screening. The cumulative dose of even this ow-dose CT scans can contribute risk although the NLST results were based on scanning annually for three years. ${ }^{14}$

Over diagnosis, an inherent part of any screening program is the detection of indolent or occult disease that would not otherwise have become clinically significant or impacted patient outcome. Downside of over diagnosis may cause unnecessary morbidity, mortality, anxiety, cost, and labels a patient with a disease. ${ }^{17,18}$ In the NLST, there were an excess number of lung cancers in the CT arm, compared to the chest radiograph arm. Nevertheless, over diagnosis is difficult to measure, even in a controlled trial. ${ }^{14}$ Smoking behaviors are complex, and screening is one variable in the problem. However, any LCS program should be closely affiliated with a smoking cessation program to apply this "teachable moment" and hopefully change smoking behavior in its subjects. In the NLST, $96 \%$ of the positive results in the LDCT arm were false positive. Positive screens in the majority of cases were managed with at least one follow-up CT to determine stability of the pulmonary nodules. After two rounds of screening, there are fewer false positives as a result of comparison with the baseline screening $\mathrm{CT}$, that may reveal two years of pulmonary nodule stability. Decreasing the number of false -positive lung cancer screens is an area for future research. ${ }^{14}$

\section{Discussion}

The eligibility criteria for the NLST to define high-risk persons are used in the current recommendations for LCS as the following: former or current cigarette smokers between the ages of 55 and 74 , with at least 30 pack-years smoking history. The former smokers should have quit smoking within the last 15 years. ${ }^{14}$ LDCT screening is also included in the NCCN guidelines, based on lower level evidence, in persons 50 or older, with at least a 20 pack-year smoking history, and one additional risk factor. ${ }^{12}$ LDCT screening for lung cancer is not intended for persons with clinical symptoms, such as weight loss, chest pain, or cough. Standard-dose CT with intravenous contrast-medium administration is the present standard of care in these symptomatic patients. ${ }^{14}$ The additional risk factors are a family history of lung cancer, a personal history of cancer or lung disease, occupational exposure to asbestos, silica, chromium, arsenic, cadmium, nickel, beryllium, and diesel fumes, and radon exposure that were based on the results of the International Early Lung Cancer Action program, including persons 40 years of age and older, with either a history of cigarette smoking, exposure to secondhand smoke, or occupational exposure to asbestos, radon, beryllium, or uranium. ${ }^{19}$
The number of diagnosed lung cancers per population screened would be higher, and fewer persons would be unnecessarily exposed to the related risks when eligibility criteria for lung cancer screening are further refined..$^{14}$ In this regard, additional models have been developed to calculate a person's lung cancer risk. ${ }^{20,21}$ These models have the potential of enrolment into lung cancer chemoprevention strategies in addition to having the potential of selecting patients for screening. ${ }^{20,21}$ The Spitz model and the Liverpool Lung Project (LLP) model were published in 2007 and 2008, respectively. ${ }^{20,21}$ All models include risk factors, such as age, occupational exposure, and smoking duration. The Spitz model included physician-diagnosed emphysema, and family history of cancer in first-degree relatives whereas the LLP multivariate risk model included smoking duration (never, 1-20 years, 21-40 years, 41-60 years, $>60$ years), family history of lung cancer (never, early onset $[<60$ years], late onset [ $>60$ years]), prior diagnosis of occupational exposure to asbestos, prior diagnosis of malignant tumor, and prior diagnosis of pneumonia. ${ }^{20,21}$ Risk prediction models can be assessed by several qualities, such as accuracy, clinical utility, calibration, and discrimination. Discrimination is the ability of the model to differentiate between those persons who will develop disease versus those who will not develop disease. ${ }^{22}$ Risk estimates can potentially reduce the cost of LCS by focusing those at highest risk and have been used to design large-scale randomized control lung cancer screening trials. Genetic profiles and the results of the baseline screening examination can potentiate further refining the risk modeling. Risk modeling could define the frequency of follow-up in addition to who should be screened. ${ }^{14}$

\section{Conclusion}

In the results of the NLST, around $20 \%$ reduction in lung-cancer specific mortality with LDCT screening in a high-risk population was demonstrated and contributed to a turning point in LCS. LCS with LDCT has shown that there are indolent lung cancers that may not be fatal. Further studies are urgently needed if the maximization of the risk-benefit ratio in LCS has to be achieved.

\section{Funding}

None.

\section{Acknowledgments}

None.

\section{Conflicts of interest}

Authors declare that there is no conflict of interest.

\section{References}

1. Henschke CI, McCauley DI, Yankelevitz DF, et al. Early lung cancer action project : overall design and findings from baseline screening. The Lancet. 1990;354(9173):99-105.

2. Kaneko M1, Eguchi K, Ohmatsu H, et al. Peripheral lung cancer : screening and detection with low-dose spiral CT versus radiography. Radiology. 1996;201(3):798-802.

3. Sone S, Takashima S, Li F, et al. Mass screening for lung cancer with mobile spiral computed tomography scanner. Lancet. 1998;351 (9111):1242-1245.

4. Nawa T1, Nakagawa T, Kusano S, et al. Lung cancer screening using low-dose spiral CT: results of baseline and 1-year follow-up studies. Chest. 2002;122(1):15-20. 
5. Thun MJ1, Carter BD, Feskanich D, et al. 50-year trends in smokingrelated mortality in the United States. N Engl J Med. 2013;368(4):351364.

6. Henschke CI1, Naidich DP, Yankelevitz DF, et al. Early lung cancer action project : initial findings on repeat screenings. Cancer. 2001;92 (1):153-159.

7. Sone S1, Li F, Yang ZG, et al. Results of three-year mass screening program for lung cancer using mobile low-dose spiral computed tomography scanner. Br J Cancer. 2001;84(1):25-32.

8. Swensen SJ, Jett JR, Hartman TE, et al. Lung cancer screening with CT : Mayo Clinic experience. Radiology. 2003;226(3):756-761.

9. Jaklitsch MT, Jacobson FL, Austin JH, et al. The American guidelines for lung cancer screening using low-dose computed tomography scans for lung cancer survivors and other high-risk groups. J Thorac Cardiovasc Surg. 2012;144(1):33-38.

10. Wender R1, Fontham ET, Barrera E Jr, et al. American Cancer Society lung cancer screening guidelines. CA Cancer J Clin. 2013;63(2):106117.

11. Wood DE, Kazerooni EA, Baum SL, et al. Lung Cancer Screening, Version 3.2018, NCCN Clinical Practice Guidelines in Oncology. J Nat Compr Canc Netw. 2012;10(2):240-265.

12. Bach PB1, Mirkin JN, Oliver TK, et al. Benefits and harms of screening for lung cancer : a systematic review. JAMA. 2012;307(22):2418-2429.
13. Caroline C. Lung cancer screening with low-dose CT. Radio Clin North Am. 2014;52(1):27-46.

14. Brenner DJ. Radiation risks potentially associated with low-dose CT screening of adult smokers for lung cancer. Radiology. 2004;231(2):440 445 .

15. Brenner DJ, Hall EJ. Computed tomography : an increasing source of radiation exposure. $N$ Engl J Med. 2007;357(22):2277-2284.

16. Bley A, Welch HG. Effect of three decades of screening mammography on breast-cancer incidence. $N$ Engl J Med. 2012;367(21):1998-2005.

17. Veronesi G, Maisonneuve P, Bellomi M, et al. Estmating overdiagnosis in low-dose computed tomography screening for lung cancer: a cohort study. Ann Intern Med. 2012;157(11):776-784.

18. International Early Lung Cancer Action Program Investigators, Henschke CI, Yankelevitz DF, et al. Survival of patients with stage I lung cancer detected on CT screening. N Engl J Med. 2006;355(17):17631771 .

19. Spitz MR, Hong WK, Amos CI, et al. A risk model for prediction of lung cancer. J Natl Cancer Inst. 2007;99(9):715-726.

20. Cassidy A, Myles JP, van Tongeren M, et al. The LLP risk model an individual risk prediction model for lung cancer. $\mathrm{Br} J$ Cancer. 2008;98(2):270-276.

21. Etzel CJ, Bach PB. Estimating individual risk for lung cancer. Semin Respir Crit Care Med. 2011;32(1):3-9. 